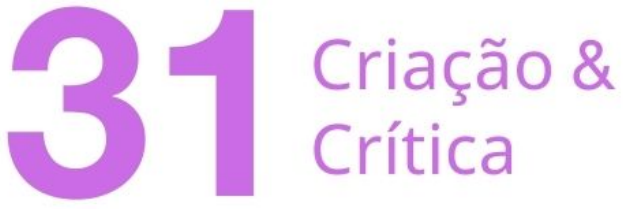

\section{LA MÉLODIE FRANÇAISE COMME TÉMOIGNAGE D’UNE RÉCEPTION : LE CAS D'INVOCATION}

Lucia Pasini ${ }^{1}$

\begin{abstract}
RÉSUMÉ : Tout au long de cet article, j'esquisse en première instance les contours d'un appareil conceptuel afin de définir les termes dans lesquelles je situe mon travail, et je montre ensuite par le biais de l'exemple d'Invocation à quel point la prise en compte du genre mélodique dans une optique littéraire s'avère fructueuse. L'analyse de cet exemple est à son tour divisée en deux parties : une première où il est question de situer la mélodie dans son contexte de production, et une deuxième où l'on se tourne effectivement vers le morceau et ses caractéristiques particulières. En proposant un appareil conceptuel pour concevoir littérairement la mélodie française, je m'appuie sur trois pôles théoriques de références, dont la provenance est hétérogène, à savoir : la théorie de la réception; la notion de " fenêtre herméneutique », telle que la présente Lawrence Kramer ; et la notion de "réseau des textes possibles », telle qu'en parle Michel Charles. Ensuite, je présente un exemple isolé pour montrer le potentiel d'une conception littéraire de la mélodie française comme témoignage de la réception par les compositeurs des textes poétiques qu'ils choisissent pour leurs morceaux.
\end{abstract}

MOTS-CLÉS : Coppée, Cazaneuve, mélodie française, réception, herméneutique, interprétation

\section{THE MÉLODIE FRANÇAISE AS EVIDENCE OF A RECEPTION PROCESS THE CASE OF INVOCATION}

\begin{abstract}
In this article, I begin by sketching a few notions in order to define the terms of my work, then I show, through the example of Invocation, how the literary conception of the mélodie française is productive. The analysis of this example is divided in two parts: in the first, the mélodie is situated in the context of its production, and, in the second, we move to the actual piece and its peculiarities. While proposing the bases for my perspective, I describe three main points of reference: the theory of reception, the notion of "hermeneutic window" presented by Lawrence Kramer, and the notion of "web of possible texts", proposed by Michel Charles. Then, I present an isolated example to show the potentialities of the literary conception of the mélodie française - a conception that sees it as the expression of the reception by the composers of the poems they choose for their pieces.
\end{abstract}

\footnotetext{
${ }^{1}$ Lucia Pasini est doctorante en Littérature française et comparée à l'Université Sorbonne Nouvelle, où elle est membre du laboratoire CRP19. Son doctorat se déroule dans le cadre d'une cotutelle avec le Dottorato in Lettere de l'Université de Turin. Sa thèse porte sur la mélodie française et la réception musicale des poètes français dans la période entre 1870 et 1914, et elle est dirigée par Cécile Leblanc et Andrea Malvano. Email : lucia.pasini@sorbonne-nouvelle.fr.
} 


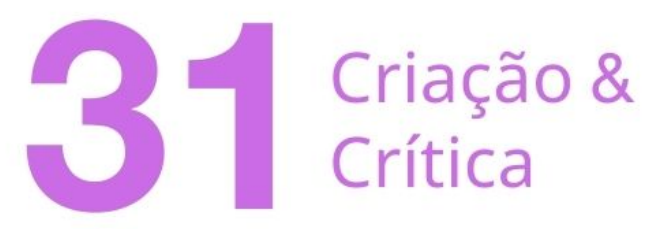

KEYWORDS: Coppée, Cazaneuve, mélodie française, reception, hermeneutics, interpretation

\section{Introduction}

«La mélodie française se situe en fait dans le cœur incandescent d'une série d'enjeux concernant le langage et la signification » (ANGER, 2015) : ce sont les mots de Violaine Anger sur ce genre musical qui englobe les mises en musique des textes conçus indépendamment de l'intervention possible d'un compositeur. L'on considère conventionnellement l'année 1831 comme la date de naissance du genre, lorsque Berlioz compose ses Mélodies irlandaises, mais la production mélodique continue son parcours artistique jusqu'à nos jours ${ }^{2}$. Dans ce qui suit, je me propose de concevoir la mélodie française comme le témoignage de la réception par les compositeurs des poèmes mis en musique. Ce témoignage se manifeste premièrement dans le choix d'un texte plutôt que d'un autre, mais il s'articule plus précisément à l'intérieur des morceaux, où chaque choix expressif contribue à transmettre à l'auditeur une vision particulière du poème.

Plus précisément, la mélodie (mais aussi n'importe quelle mise en musique d'un texte, soit-elle Lied, chanson, etc.), en tant qu'objet artistique hybride, pose une énorme quantité de problèmes d'ordre esthétique, sémantique, et réceptif. En effet, la relation esthétique entre l'instance productrice de la signification (tout premièrement le poète, mais aussi le «je lyrique ", et dans ce cas le compositeur et l'exécuteur ${ }^{3}$ également) et l'instance qui le reçoit (le lecteur ou le spectateur ou l'auditeur) est problématisée de façon plutôt évidente par la multiplication des facteurs impliqués - dans les mots de Michel Imberty : «l'interprétation est en fait une interaction complexe entre l'œuvre écrite, le projet compositionnel d'un compositeur et la compréhension personnelle qu'en a l'interprète, sans compter ce que l'auditeur de cet interprète peut ajouter à cet échafaudage déjà bien chargé ${ }^{4}$ (IMBERTY, 1992, p. 41). Évidemment, toutes ces questions doivent être prises en compte. Pour le faire, il est nécessaire de convoquer les instruments de l'analyse littéraire et ceux de l'analyse musicale, et de les employer conjointement. Mon but est donc assez proche de celui de Michel Gribenski, qui s'exprime ainsi à propos de son entreprise ${ }^{5}$ (GRIBENSKI, 2004, p. 129) :

\footnotetext{
2 V. sur ce sujet Faucher, 2010.

${ }^{3}$ Je dis exécuteur, au lieu d'interprète, pour ne pas engendrer de la confusion par rapport à la double signification du mot interprète, à savoir justement exécuteur et herméneute.

${ }^{4}$ Toutefois, un peu plus loin dans ce même texte, Imberty relate une expérience conduite sur la mélodie Mandoline de Debussy sur un poème de Verlaine : « J'avais donc demandé aux interprètes d'enregistrer deux versions de Mandoline, l'une dans un tempo deux fois plus lent que l'autre qui respectait les indications de Debussy. Le résultat essentiel de cette expérience a été que ce ralentissement de tempo ne modifie pas sensiblement le climat émotionnel et expressif de la pièce qui tient à bien d'autres facteurs propres à l'écriture, et indépendants de l'interprétation elle-même " (IMBERTY, 1992, p. 4243). Par conséquent, il serait prudent de nuancer la portée de l'influence que l'exécution peut avoir sur la perception des auditeurs ; de l'autre côté, l'expérience d'Imberty n'interroge que les changements de tempo, et l'on peut imaginer que la modification d'autres paramètres saillants pourrait entraîner des résultats différents.
}

${ }_{5}^{5}$ Pour en savoir plus, v. Gribenski, 2008. 


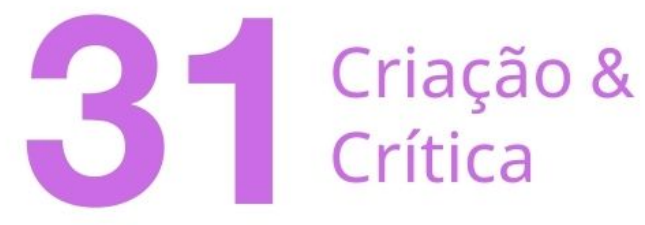

Dans une perspective résolument interdisciplinaire, sont convoquées à la fois la linguistique, la métrique, et la musicologie sous son double aspect analytique et historique. Mais plus encore qu'interdisciplinaire, ce travail se veut comparatiste. Comparer, c'est non seulement faire apparaître des similitudes et des différences, mais, de façon beaucoup plus dynamique, mettre en relation, c'est-à-dire en tension, pour faire surgir des spécificités. Or, il s'agit bien, en effet, de faire jouer, l'une par rapport à l'autre, deux formes d'expression (musique et littérature, à la fois arts et langages) [...] - il s'agit donc bien, au sein même d'un corpus musico-linguistique donné, de confronter plusieurs lectures musicales d'un même texte, avec le souci de ne pas se contenter de jugements normatifs, mais de mettre au jour la pluralité des choix de lecture.

Je souscris largement à ce propos, mais je souligne également que sa recherche " vise à mettre à jour les relations entre rythmes verbal et musical à la fin du XIX ${ }^{\text {e }}$ siècle, en France et en Allemagne, à travers les réalisations ou performances qu'en offrent la diction et la prosodie musicale " (GRIBENSKI, 2004, p. 112), ce qui la situe transversalement par rapport à la mienne dans le sens où, si elle s'étend jusqu'en Allemagne et au répertoire opératique, elle se concentre spécifiquement sur les questions de la prosodie musicale, alors que celles-ci ne sont qu'une partie de ma perspective.

Pour illustrer cette perspective je me propose de me pencher sur la mélodie Invocation, parue en 1879 chez Henri Tellier, à l'intérieur du recueil Douze mélodies pour chant et piano ${ }^{6}$, du compositeur Édouard Cazaneuve sur un texte de François Coppée. Pourquoi choisir une mélodie d'un auteur qui a été oublié au fil du temps ? La raison en est simple. Lorsque l'on pense à la mélodie française, l'on se tourne d'habitude vers les quelques grands noms qui ont donné à ce genre du lustre et du prestige, surtout vers la fin du XIXe siècle et le début du XXe à savoir Fauré, Debussy, Massenet, Duparc et Gounod. Ces auteurs ont en effet joué un rôle d'importance capitale pour façonner le genre de la mélodie, grâce aux nombreux morceaux proéminents qu'ils ont composés et aux également nombreuses exécutions publiques de ces morceaux. Toutefois, il ne faut pas oublier que la grande majorité de la production mélodique n'a jamais vu la salle de concert : il s'agit d'un genre dédié au salon, à l'exécution par les amateurs. Par conséquent, il me semble intéressant de me tourner vers l'un de ces pièces oubliées, mais qui se proposent comme la production mélodique moyenne, pour ainsi dire.

Tout au long de cet article, par conséquent, j'esquisserai en première instance les contours d'un appareil conceptuel afin de définir les termes dans lesquelles je situe mon travail, et je montrerai ensuite par le biais de l'exemple d'Invocation à quel point la prise en compte du genre mélodique dans une optique littéraire s'avère fructueuse. L'analyse de cet exemple sera à son tour divisée en deux parties : une première où il sera question de situer la mélodie

${ }^{6}$ Celles-ci sont digitalisées et disponibles dans Gallica : https://gallica.bnf.fr/ark:/12148/bpt6k321821w. Accès en : 15 août 2021. 


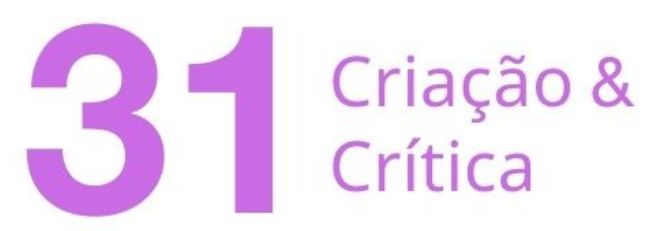

dans son contexte de production, et une deuxième où l'on se tournera effectivement vers le morceau et ses caractéristiques particulières.

\section{L’appareil conceptuel}

En proposant un appareil conceptuel pour concevoir littérairement la mélodie française, je m'appuie sur trois pôles théoriques de références, dont la provenance est hétérogène, à savoir : la théorie de la réception ; la notion de « fenêtre herméneutique ", telle que la présente Lawrence Kramer ; et la notion de "réseau des textes possibles », telle qu'en parle Michel Charles. Je voudrais également souligner, en début d'exposition, que ce qui suit est pensé justement comme un panorama d'outils conceptuels qui justifient une conception de la mélodie française en tant que le lieu d'une réception proprement littéraire, et que, par conséquent, il est question ici non pas d'une véritable méthodologie mais plutôt d'une esquisse théorique. Celle-ci se trouve évidemment en dialogue avec la pratique analytique et interprétative, mais ce n'est pas mon intention à ce stade d'établir un programme avéré.

Dans le contexte contemporain, la théorie de la réception est largement connue (JAUB, 1978 ; ISER, 1985 ; CHARLES, 1977). Selon cette conception de la relation esthétique entre l'artiste et le récepteur, ce dernier joue un rôle actif dans la constitution de l'objet artistique en question. Dans ce cadre, le processus de mise en musique d'un texte peut être considéré comme un travail herméneutique, plus ou moins conscient, plus ou moins approfondi, mais qui mérite d'être pris en considération dans le domaine des études littéraires. En outre, l'étude de la réception des poèmes aussi bien que celle des mélodies elles-mêmes permet d'aboutir à une compréhension raffinée des relations communicationnelles parmi les pôles du poète, du compositeur, des exécuteurs, et du public - bref, des instances mentionnées plus haut comme responsables, dans leur interaction, de la négociation de la signification. II faut tout de même garder à l'esprit que cette négociation est spécifiquement déterminée par l'espace social et/ou géographique, tout comme par le moment de l'histoire, où elle a lieu. L'étude de la réception pourra donc éclaircir les diverses façons dont les artefacts en question sont appréhendés comme significatifs par des communautés interprétatives différentes, mais qui interagissent, se superposent et se transforment au fil du temps.

La notion de " communauté interprétative » a été proposée par Stanley Fish (1976), et elle a été développée subséquemment (FISH, 2007). Selon le critique, "les significations ne sont la priorité ni de textes stables et fixes ni de lecteurs libres et indépendants, mais de communautés interprétatives qui sont responsables à la fois de la forme des activités d'un lecteur et des textes que cette activité produit» (p. 55). Dans ce contexte, la communauté interprétative constituée par les compositeurs peut être considérée comme un endroit privilégié pour la réception des textes poétiques, vu que le compositeur est un lecteur compétent, habitué à lire de la poésie, mais qui n'est ni un technicien de la versification ni un lecteur professionnel. Et, du côté de la réception des mélodies, s'il est vrai que le concept de communauté interprétative a été élaboré à partir des notions établies dans le but d'une application aux textes littéraires, il me semble qu'elle pourrait être appliquée avec un succès égal dans le domaine des études musicologiques, même si le décantage de cette notion cause une complication, notamment due à la multiplication des facteurs convoqués dans la réception 


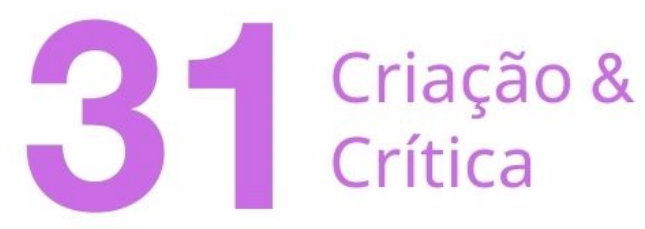

musicale, dont il a été question plus haut. II faut bien entendu tenir compte de cette complication, qui a d'ailleurs été l'objet des études sur la performance?

II est évident que, dans ces circonstances, l'approche structuraliste à l'analyse littéraire doit être rejetée : de fait, en abolissant non seulement l'auteur, mais aussi le lecteur et le contexte en tant qu'entités historiquement déterminées, cette approche va essentiellement à l'encontre des présupposés que l'on vient d'énoncer. Par conséquent, elle ne peut pas trouver sa place dans une étude qui, comme celle-ci, se propose de rester constamment vigilante par rapport à la réalité historique de son objet.

Ensuite, Lawrence Kramer (2006) a proposé la notion de « fenêtre herméneutique », comprise comme l'endroit où l'objet de l'interprétation apparaît comme explicitement problématique. Selon sa formulation, « l'interprétation s'envole à partir des points de rupture, qui apparaissent habituellement comme des points de sous- ou de sur-détermination : d'un côté, un créneau, un manque, un lien défaillant ; de l'autre, un excès dans le dessin, une répétition supplémentaire, un lien excessif $»^{8}$ (p. 12). L'identification, dans un premier moment, et l'analyse, ensuite, de ces endroits de problématisation explicite apporte non seulement une compréhension hyper-localisée de ces points spécifiques, mais informe aussi la compréhension générale du morceau, qui se trouve changée par la prise en compte des interactions entre les différentes composantes convoquées dans l'enquête des fenêtres herméneutiques.

Kramer prend ouvertement en compte la possibilité d'appliquer cette notion à l'étude de la musique accompagnée d'un texte (p. 13), mais sa préoccupation principale demeure la musique instrumentale. Son insistance sur la pertinence de l'approche herméneutique est en partie justifiée précisément par ce souci : l'herméneutique en tant que discipline a ses origines dans l'étude des textes, alors que, historiquement, l'analyse musicale a tendance à rejeter des approches qui l'emmènerait hors d'elle-même. En effet, Kramer souligne que, s'il a choisi de nommer cette notion « fenêtre herméneutique ", c'est justement pour « s'opposer à l'idée qui voit la musique comme purement suffisante à elle-même, comme autoréférentielle, comme une monade sans fenêtres " $^{9}$ (KRAMER, 2012, p. 68).

Or, la conception de la musique que Kramer propose ne va pas de soi : le débat sur la signification de la musique, qu'elle soit définie tour à tour comme référentielle, analogique, métaphorique, émotionnelle, intrinsèque, voire inexistante - ce débat n'est pas nouveau. On pourrait le résumer en trois positions principales, même si, à l'intérieur de ces positions, il est possible de nuancer considérablement les stances de chaque penseur : la position formaliste qui défend l'autonomie de la musique ; la position sémiologique, qui comprend la musique comme fondamentalement symbolique ; et la position socio-historique, si l'on veut, qui voit la musique comme ancrée dans son contexte social et historique, justement. Ces deux dernières stances sont quelque peu superposées, puisque les symboles musicaux enquêtés par la

\footnotetext{
7 Je ne m'arrête pas sur ce point ici pour des raisons d'espace, mais je renvoie à Borio et Garda (1996). 8 «Interpretation takes flight from breaking points, which usually means from points of under- or overdetermination : on the one hand, a gap, a lack, a missing connection ; on the other, a surplus of pattern, an extra repetition, an excessive connection ».

${ }_{9}$ " One reason I call these points hermeneutic windows is to counter the idea of music as purely selfsufficient and self-reflective, a windowless monad ».
} 


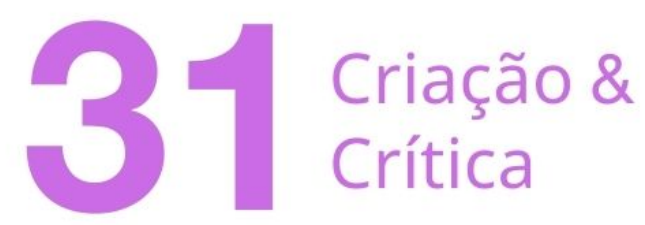

sémiologie renvoient, en tant que symboles, à quelque chose d'autre que la musique ellemême, et ce quelque chose pourrait bien être une réalité sociale ou historique.

Le formalisme musical a été défendu en particulier par Eduard Hanslick (1865) : il propose une conception selon laquelle la musique signifie sans convoquer des éléments externes à elle-même, soient-ils de caractère associatif ou émotionnel. Ce propos, tel que Hanslick le formule, est plutôt tranchant et devient problématique lorsque l'on essaie de l'appliquer systématiquement. Toutefois, il serait imprudent de le rejeter complètement. En effet, au cours du $X X^{e}$ siècle, il a donné lieu à des réflexions qui me paraissent tout à fait partageables, comme celles de Leonard B. Meyer (1956 ; 1973). Selon ses formulations, un auditeur expérimenté dans un certain style musical comprend des motifs ou des événements musicaux comme suggérant ou impliquant des modalités de continuation ou d'élaboration spécifiques à l'intérieur du morceau. Ceci renvoie ouvertement à la position formaliste, puisque, dans ce cadre, la perception musicale est déterminée par la structure et par la forme. Or, vu que chaque analyse musicale a la prétention de décrire son objet comme il serait entendu par un auditeur imaginaire, c'est exactement le prototype de l'auditeur savant proposé par Meyer qui sera pris comme point de référence dans cette étude. En ce sens, ce type de formalisme modéré, pour ainsi dire, doit être retenu : d'un côté, il n'empêche pas l'intégration d'autres modalités (externes) de signification de la musique, et, de l'autre, il serait incorrect, me semble-t-il, de nier toute pertinence à la structure formelle du morceau dans une enquête sur la signification musicale. Pour ces raisons, dans le contexte de l'analyse et quand ceci s'avérera pertinent, cette structure formelle sera décrite dans son ensemble, à un niveau macro-structural, mais aussi à un niveau plus local, notamment celui de l'harmonie fonctionnelle, puisque celle-ci est bien le langage employé dans Invocation.

De son côté, la sémiologie musicale trouve ses origines dans l'œuvre de Susanne Langer (1942), qui s'est engagée dans la construction d'une théorie de l'intelligence humaine en tenant compte de l'importance cognitive des pratiques artistiques, et qui a exercé une influence immense sur la réflexion philosophique autour de la signification de la musique. A partir de ses réflexions une conception qui voit la musique comme symbolique a pu être défendue ${ }^{10}$ : en France, cette lignée sémiologique a bien été représentée par Jean-Jacques Nattiez (1987) et, plus récemment, par Marta Grabocz (2007 ; 2009). Tout comme dans le cas de l'approche formaliste, l'approche sémiologique ne peut pas être rejeté dans son ensemble : il est effectivement indéniable que certains traits musicaux soient symboliques. Par exemple, l'on peut penser aux mises en musique de la troisième "Ariette oubliée » de Paul Verlaine (1891, p. 6-7) par Debussy (1903) et Fauré (1888), qui intitule la sienne Spleen. Or, comme l'on sait, la pluie est un motif récurrent dans le poème, qui place une certaine emphase sur l'effet que le bruit de la pluie cause dans le cœur de celui qui parle. De même, on peut reconnaître dans les croches (chez Fauré) et les doubles-croches (chez Debussy) au piano une représentation symbolique de ce bruit. Cet exemple montre bien l'efficacité de l'approche sémiologique, qui permet promptement de voir dans ce trait musical un topos de la représentation de la pluie, mais il montre également ses limites : en effet, une fois que l'on a reconnu cette représentation, on ne sait rien sur les raisons qui justifient sa présence ou sur

10 Pour des références anglophones, voir Ratner (1980), Agawu (1991), et Hatten (1994). 


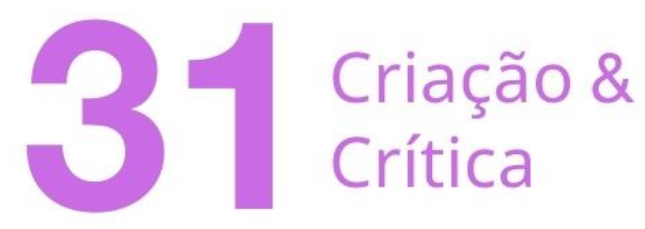

ses conséquences sur la façon dont le morceau dans son entièreté pourra être perçu ${ }^{11}$. (Dans ce cas, ces raisons sont plutôt évidentes : il s'agit d'une imitation musicale de la pluie évoquée dans le texte.)

Et finalement, l'approche socio-historique est bien celle de Kramer, que l'on a décrit plus haut, et celle qui informe cette étude et ses présupposés principaux. Pour résumer, donc, la notion de fenêtre herméneutique présente plusieurs avantages : tout d'abord, elle permet de concevoir la musique comme une réalité historique, ancrée dans son contexte social en ce qui concerne sa production, son exécution et sa réception ; deuxièmement, elle permet de convoquer des approches différentes à l'analyse musicale, comme par exemple l'approche formaliste ou sémiologique, alors que celles-ci deviennent appropriées pour expliquer un trait particulier de l'objet de l'analyse ; et enfin, elle exclue toute question non pertinente, vu que son point de départ est le morceau musical.

Le troisième point de référence que j'ai évoqué au début de cette partie est à rechercher dans les mots de Michel Charles (2010, p. 389-390) :

L'ensemble de ce que l'on appelle les lectures d'un texte sera défini comme un ensemble de textes appartenant à un même réseau. Plus précisément, si l'on considère tout texte comme une collection d'énoncés, on posera que le réseau en question est un réseau d'énoncés, qu'une multitude de textes peuvent être construits en reliant ces énoncés, et que les lectures renvoient de l'un à l'autre, les connectant de proche en proche au prix de multiples transformations et variations. Le texte, ou ce qu'on nomme communément « le texte », sera donc ultimement un réseau textuel qui se monnaie en détail pour donner une multitude de textes possibles qu'actualisent les interprètes.

Le théoricien esquisse ici une conception du texte et de sa réception qui promet d'être fructueuse dans le cadre de cette étude. Tout d'abord, il souligne comment un texte peut être compris de plusieurs façons différentes et qu'il serait futile de classer ces interprétations multiples en fonction de leur degré de vérité : en effet, si l'on peut réfuter une interprétation fausse, il est impossible de prouver définitivement la justesse d'une interprétation valide lorsqu'elle est confrontée par une autre interprétation valide. Tout de même, il est important de souligner que cette pluralité ne permet nullement de dire n'importe-quoi à propos d'un texte donné : c'est le texte lui-même qui donne des limites à l'herméneute, au-delà desquelles l'interprétation devient fautive.

Ensuite, cette conception du réseau des textes possibles pose une tâche devant l'analyste (CHARLES, 2010, p. 389-390) :

${ }_{11}$ Boucourechliev (1993) décrit ce qu'il appelle une « sémantique musicale élémentaire » (p. 10) et, même s'il n'emploie pas de termes renvoyant à la sémiologie, il me semble que c'est bien à ce type d'approche qu'il fait référence. II s'exprime également sur le poids à donner aux considérations fondées sur cette perspective : « on voit à quel niveau il convient de situer cette sémantique qui [...] ne saurait, en tout cas, être confondue avec le sens » (p. 11). 


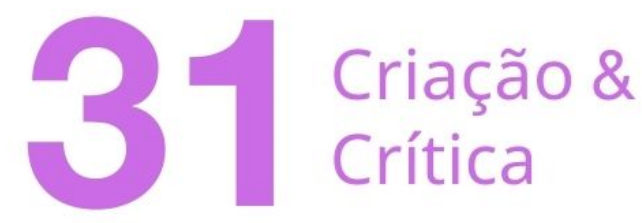

II est légitime de penser que l'analyse gagnera considérablement à se préoccuper de cet objet étrange qu'est le réseau textuel. S'intéresser à l'ensemble des textes qu'il est possible de construire, c'est en effet s'intéresser aussi, et peut-être surtout, à leur articulation, à la manière dont les énoncés qui les constituent sont reliés dans la constitution du réseau ; c'est étudier l'architecture même de ce réseau, ses nœuds, ses bifurcations, ses connexions, ses équilibres provisoires; c'est analyser les modes de coexistence des textes possibles, leurs superpositions, les mécanismes de substitution, les effets de mémoire et d'oubli. [...] On peut dès lors s'intéresser au filtre herméneutique du critique : quel type de regard lui a permis de construire le texte qu'il a construit (la problématique est connue), soit aux énoncés qui, dans le réseau, ont été accentués, à ceux qui sont restés dans l'ombre, à ceux qui ont été déplacés, bref à ce qui a rendu possible, justement, l'élaboration de tel texte. C'est en effet dans la mouvance de la réception active que s'esquissent les textes possibles, cette mouvance qu'il convient en premier lieu d'essayer de décrire.

C'est exactement cette description que j'envisage d'entreprendre, mais il faut remarquer qu'une analyse de ce type demande plusieurs passages. Tout d'abord, il faut justement comprendre l'articulation du réseau d'énoncés qui constitue le texte. Pour le faire, on doit convoquer les instruments habituels de l'analyse littéraire, à commencer par l'analyse linguistique des relations syntagmatiques et paradigmatiques, et, dans le cas d'un poème comme Invocation, métriques. Ensuite, l'on décrira quelques exemples de textes possibles, et l'on mettra en évidence les liens et les oppositions entre les différents pôles du réseau, tout en gardant à l'esprit la relation aux énoncés textuels de départ, à ceux qui « ont été accentués, à ceux qui sont restés dans l'ombre, à ceux qui ont été déplacés ".

Toutefois, je voudrais avancer encore un peu par rapport aux propositions de Charles : une fois décrit ce réseau des textes possibles, l'on devra le confronter à la mise en musique. Le présupposé ici est double : d'un côté, la mise en musique se situe elle-même dans le réseau des textes possibles, en tant qu'interprétation du texte poétique ; de l'autre, la mise en musique doit être interprétée à son tour avant de pouvoir être intégrée dans le réseau. Par conséquent, l'analyse proprement dite de la mélodie doit être accompagnée d'une comparaison constante aux articulations du réseau textuel : par exemple, si l'on a constaté dans l'étude du réseau que la mise en évidence d'un certain énoncé aboutit à une certaine compréhension du texte, l'on pourrait avancer que la mise en évidence du même énoncé dans la mélodie (par d'autres moyens, évidemment) signale que le compositeur a compris le texte d'une façon similaire. Si cette démarche est quelque peu risquée, l'on serait peut-être plus à l'aise en déterminant les façons dont le compositeur n'a pas compris le texte. En tout cas, le but du processus demeure le même : situer la mise en musique dans le réseau des textes possibles engendré par le poème qu'elle prend comme source. 


\section{Criação \&}

\section{INVOCATION DANS SON CONTEXTE}

Avant d'entreprendre l'analyse proprement dite, il faut tout d'abord s'interroger sur la place d'Invocation dans les productions respectives de Cazaneuve et de Coppée. Je commence par ce dernier, pour passer au premier dans un deuxième moment.

François Coppée a laissé des traces nombreuses dans la mémoire collective de ses contemporains. Né en 1842, la même année que Mallarmé et José-Maria de Heredia, Coppée se lie dans un premier moment au cercle parnassien, dont font partie Sully Prudhomme, Léon Dierx, Armand Silvestre, Catulle Mendès, Anatole France, Louis Xavier de Ricard, Leconte de Lisle tout comme Paul Verlaine et Théodore de Banville. C'est dans ce même milieu, et surtout grâce à son amitié avec Verlaine, qu'il fait la connaissance d'Emmanuel Chabrier au début des années $1860 \mathrm{~s}^{12}$. En 1871, à la suite du succès du Passanten $1869^{13}$, un autre compositeur célèbre, Henri Duparc, met en musique son poème La Vague et la cloche, mais cette mélodie ne sera publiée qu'en 1894 par Rouart, Lerolle et Cie (DARCOURS, 1894). Quelques années plus tard, en 1875-76, lorsque le troisième recueil du Parnasse contemporain est en préparation, il fait partie du jury chargé du choix des poèmes, à côté de Banville et d'Anatole France (POPOVIC, 1993, p. 108-109). Le recueil qui contient Invocation, intitulé L'Exilée, paraît l'année suivante, en 1877. En 1884, il entre à l'Académie française et continue à publier des recueils de vers jusqu'en 1906, deux ans avant sa mort.

A partir d'une étude sur la nature des alexandrins présents dans ses recueils, JeanMichel Gouvard esquisse une répartition en quatre segments de la production de Coppée (GOUVARD, 1994) :

1. «jusqu'en 1868, époque de la jeunesse et, entre autres, de l'amitié et de la collaboration avec Verlaine », quand « environ 3 alexandrins du poète sur cent sont a-classiques »;

2. les deux années qui suivent, quand «moins de deux sur cent présentent un marquage anté-césural »;

3. de 1870 à 1875 , quand « il faut lire près de 200 alexandrins pour en trouver un marqué »;

4. après 1875 et jusqu'à la mort du poète, quand « ce sont 250 vers qu'il est nécessaire de parcourir pour rencontrer une occurrence CPM6 $»^{14}$.

Comme on le voit, Gouvard remarque une progressive normalisation des alexandrins du poète, qui deviennent de plus en plus réguliers au fil du temps. Cette trajectoire inverse à

12 Chabrier notera sur son exemplaire de Jadis et naguère : «Pendant 2 ou 3 ans, de 1860 à 1863, rue Lécluse, aux Batignolles, j'allais diner chez Madame Verlaine, presque tous les samedis [...]. Étaient commensaux Mérat, Racot, Coppée, L.X. de Ricard, Ed. Lepelletier » (cité dans DELAGE, 1994, p. 8). L'on sait en outre que Coppée fréquente également Théophile Gautier, les frères Goncourt et Théodore de Banville (cf. CONDEMI, 1992, p. 124).

${ }^{13}$ Dans les mots de Steve Murphy (1996, p. 255) : « Coppée [...] allait bientôt être considéré comme l'une des étoiles montantes de la poésie française, mais ce fut surtout après le succès du Passant en 1869 ». Le Passant, comédie en un acte en vers, " avait été jugée digne d'intérêt par la famille impériale et jouée aux Tuileries le 14 janvier 1869 »(MURPHY, 2000, p. 190).

${ }_{14}$ La sigle CPM6 fait référence au système d'analyse métricométrique développé par Benoît de Cornulier $(1982 ; 1995)$. 


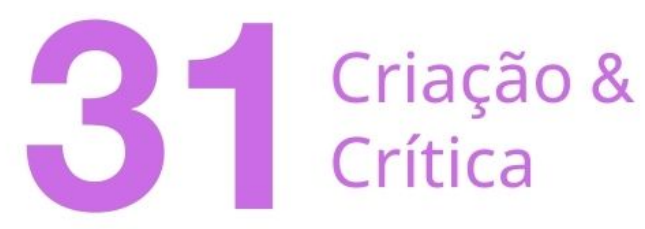

celle de la production poétique fin-de-siècle dans son ensemble s'explique par le désir de Coppée d'entrer à l'Académie et «puis, y étant entré, de se comporter en Immortel » (GOUVARD, 1994, p. 93), ce qui réclame une versification parfaite.

Ce petit résumé de la vie et de la carrière poétique de Coppée sera suffisant pour le moment ${ }^{15}$, et l'on peut maintenant se tourner vers le compositeur.

Contrairement au cas coppéen, on ne sait pas grand-chose à propos d'Édouard Cazaneuve, mais il est possible de reconstruire, au moins partiellement, quelques repères à propos de sa trajectoire à l'intérieur du milieu musical français à partir des quelques informations qui ont survécu jusqu'à aujourd'hui. Sa date de naissance est l'un des éléments qui ne sont malheureusement pas à disposition. Toutefois, l'on sait que sa première publication date de $1859^{16}$ (CAZANEUVE, 1859), et qu'il est mort en 1903. Un regard jeté sur les sections musicales dans la presse de l'époque ${ }^{17}$ permet d'ajouter des détails à ce mince portrait.

En 1863, l'auteur est nommé comme pianiste dans deux publications (BOTTE, 1863, p. 147 ; ANONYME, 1863, p. 53). Ensuite, vers la fin des années 1870s, Cazaneuve est mentionné dans quelques journaux comme " un jeune musicien au plus grand avenir ${ }^{18}$ et, plus tard, comme professeur du cours d'ensemble instrumental à l'École classique de musique et de déclamation de la rue Charras (ANONYME, 1892, p. 7-8). On annonce également un opéra-comique dont la première audition est attendue au Théâtre-Lyrique ${ }^{19}$, et l'acceptation de la part de l'Opéra-Comique de "trois actes de sa composition intitulée le Carrosse du gouverneur. [...] Le livret est de M. Cormon ${ }^{20}$ (KRAN, 1881, p. 4). En 1888, un « fort joli

15 Je signale, pour ceux qui souhaitent s'informer ultérieurement sur le poète, le volume de Léon le Meur (1932). Deux études plus brèves, mais plus récentes, sont Lefrère et Piersens (2003), et Picornot (2010. Je mentionne enfin l'entrée biographique assez brève que donne Verlaine à propos de son collègue (VERLAINE, 1905), tout comme l'édition critique de ses écrits en prose (COPPÉE, 2003).

${ }^{16}$ Le numéro d'opus présent dans cette publication pourrait être pris comme un indice utile à tracer la production du compositeur au fil des années. Malheureusement, ceci s'avère une tâche assez complexe: dans WorldCat, après l'op. 1, on rencontre tout de suite l'op. 17, publié en 1863 (CAZANEUVE, 1863). Ensuite, on se heurte aux opus de 45 à 48 en 1872 (CAZANEUVE, 1872a, $1872 \mathrm{~b}, 1872 \mathrm{c}$ ). Puis, les numéros d'opus disparaissent, même si la production s'intensifie, surtout pendant les années 1890s. Si l'on accepte donc le présupposé que certains de ces ouvrages sont probablement perdus aujourd'hui, l'on pourrait avancer provisoirement l'hypothèse, à partir de la distribution des ouvrages connus et des numéros d'opus, que, en 1902 (une année avant sa mort), Cazaneuve aurait atteint le numéro d'opus 190, au moins. Ce nombre en tant que tel est probablement fautif, mais sa grandeur considérable indique que l'auteur a été vraisemblablement un compositeur prolifique, ce qui est confirmé par le catalogue de la Bibliothèque nationale de France, où l'on peut trouver plus de 300 ouvrages à son nom.

${ }_{17}$ En guise d'avertissement, il faut signaler au lecteur que le $\mathrm{M}$. Cazaneuve le plus présent dans la presse fin-de-siècle est le commissaire de police du quartier de la Madeleine, le frère du compositeur.

18 "A la récente inauguration du nouveau théâtre des Nouveauté. Mlle Lina Bell a chanté une composition due à un jeune musicien du plus grand avenir : M. Édouard Cazaneuve. [...] C'est la célèbre Aubade, de Victor Hugo, que M. Cazaneuve a mise en musique. [...] La mélodie de M. Cazaneuve est d'une poésie pénétrante, et d'une allure large et simple. Rarement les vers du grand poète ont été aussi bien compris et interprétés. L'éditeur de M. Cazaneuve pour cette œuvre délicate, est M. Chatot, 19, rue Neuve-des-Petits-Champs. Je recommanderai en même temps du même compositeur l'adorable romance de Sainara, sur les paroles de M. Ernest d'Hervilly » (DANCOURT, 1878, p. 2).

${ }^{19}$ L'on sait toutefois qu'en mars 1881 aucune œuvre de Cazaneuve n'avait été jouée aux théâtres lyriques (DANCOURT, 1881, p. 3).

${ }^{20}$ Ce même opéra est annoncé par Le Ménestrel le 13 juin 1875. 


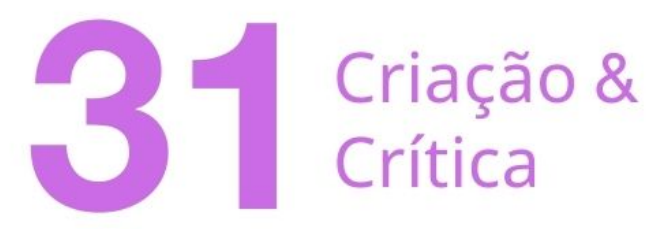

opéra-comique [...], un acte, intitulé Partie double » (THÉMINES, 1888, p. 3) est censé être terminé, mais ce travail n'est pas présenté aux directeurs de théâtre pour le moment. Par ailleurs, on ne trouve aucune trace des exécutions de ces opéras pendant les années suivantes, et l'on pourrait donc en déduire que ces travaux n'ont finalement jamais vu la scène.

En ce qui concerne les Douze mélodies pour chant et piano, dont Invocation est tirée, celles-ci apparaissent dans la « Bibliographie musicale » de La Revue et Gazette musicale de Paris du 7 mars 1880 (p. 76). Elles ont été également recensées dans La Gazette (S.B., 1880, p. 3) :

Aujourd'hui, M. Cazaneuve publie chez Tellier, 12, boulevard des Capucines (Grand-Hôtel), douze mélodies pour chant et piano, qui méritent l'attention et les suffrages de tous les dilettantes. Le choix seul des poésies sur lesquelles le compositeur a brodé ses fines mélodies, indique l'artiste de goût et le musicien de race. On lit les noms de M. Coppée [...]. Puis les noms de MM. Eugène Manuel, Armand Silvestre, Albert Delpit - dont la Ballade a été rendue par M. Cazaneuve avec un sentiment, une couleur archaïque des plus pénétrantes. [...]

Tout cela, et bien d'autres morceaux charmants, est rendu gracieusement, et parfois avec un sentiment très grave et très profond [...]. Aussi le succès de ce nouveau recueil du jeune et distingué compositeur n'a-t-il pas été un instant douteux.

Puis par Dancourt encore dans La Gazette (DANCOURT, 1881) :

J'ai déjà eu plus d'une occasion de citer avec éloges les compositions musicales de M. Édouard Cazaneuve. Ce sera, je le crois, l'un des plus distingués parmi les jeunes. II a la mélodie, une grande science et le sentiment dramatique. Les Douze mélodies nouvelles qu'il publie chez Girod (16, boulevard Montmartre) forment une gerbe lyrique dont Th. De Banville, Armand Silvestre, Coppée, J. Truffier, ont fourni le lien. Qui ne connaît la Nuit d'étoiles, de Banville [...].

M. Cazaneuve a brodé sur ces jolis vers une mélodie pénétrante et mélancolique, tout argentée de paillettes. Tout autre est la musique qui accompagne Pantomime d'Armand Silvestre et l'Aubade de J. Truffier.

Ensuite, Invocation est explicitement nommée dans la recension parue dans La Patrie du 15 novembre 1880 (THÉMINES, 1880) :

Signalons aussi une publication nouvelle de M. Édouard Cazaneuve, un mélodiste celui-ci, mais un mélodiste qui connaît son harmonie sur le bout des doigts - c'est le cas de le dire - et qui sait l'employer sans lui laisser se faire la part du lion, comme il n'arrive que trop fréquemment de nos jours. 


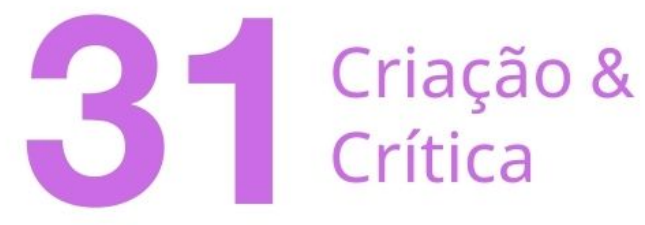

II vient de publier chez M. Tellier (ancienne maison Gérard) un recueil de Douzes [sic] mélodies nouvelles [...]. M. Cazaneuve est resté fidèle à ses poètes favoris: François Coppée, Théodore de Banville, Edmond Sylvestre [sic]. [...]

L'idylle qui ouvre le recueil et qui s'intitule Jeanne est une délicieuse pastorale [...]. Le Bonsoir de M. Sylvestre est, à coup sûr, la meilleure de ces mélodies, n'en déplaise aux autres ! parmi lesquelles il serait injuste de ne pas mentionner le Printemps de M. de Banville, et surtout l'Invocation [...] de M. Coppée : Enfant blonde aux yeux doux, ô rose de Norvège ; elle devrait être bien jolie, cette blonde sœur d'Ophélie "d'or vivant coiffée », pour que le poète lui adressât d'aussi beaux vers - il est vrai qu'il ne sait pas en faire autrement - et pour que le musicien chantât si suavement les vers du poète. J'en voudrais citer bien d'autres parmi ces Douze mélodies de M. Cazaneuve; mais je crains de faire du tort à celles que je ne nommerais pas - et que peutêtre vous aimerez davantage. Tous mes compliments à $M$. Cazaneuve.

Mais le premier commentaire paru dans les journaux semble être celui de Victorin Joncières (1879) :

Avant de clore ce feuilleton, nous voulons signaler à nos lecteurs, amateurs de chant, un recueil de douze mélodies, de $M$. Édouard Cazaneuve, sur des poésies de MM. Armand Silvestre, Eugène Manuel, François Coppée, Albert Delpit et Jules Truffier.

M. Édouard Cazaneuve a la phrase élégante et poétique, d'un tour naturel et original, exprimant avec justesse le sentiment des vers ; ses accompagnements, d'un style correct, sont intéressants sans nuire à la clarté du motif.

Nous croyons ne pas nous tromper en prédisant un vif succès dans les salons et les concerts à la Berceuse, à la Ballade, à la Chanson d'exil, à la Ritournelle, en un mot à toutes les charmantes inspirations qui composent le recueil de $\mathrm{M}$. Cazaneuve.

Je résume donc ce que l'on sait sur Édouard Cazaneuve : il publie sa première composition en 1859 ; en 1863, il joue comme pianiste en concert ; en 1879, le recueil des Douze mélodies est publié, et il reçoit des recensions favorables dans la presse ; et enfin, en 1892 , on le retrouve en guise de professeur. Ajoutons que, au fil de ces années, il compose au moins trois opéras-comiques, qui ne seront vraisemblablement jamais exécutés. Pendant la période immédiatement subséquente et jusqu'à la mort du compositeur, il n'y a guère d'informations à propos de ses occupations. Toutefois, l'on peut compléter le cadre par une dernière précision: I'on aura remarqué que Cazaneuve est souvent décrit comme « mélodiste » et son public désigné comme composé d'« amateurs » et de « dilettantes » dans 


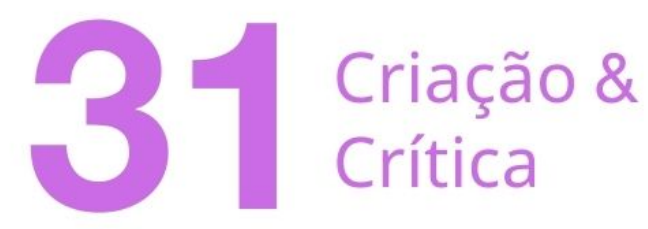

les commentaires cités plus haut. Par conséquent, l'on pourrait imaginer que le succès (relatif) de Cazaneuve était dû probablement à ce type de morceaux de chambre, destinés au marché privé des amateurs et des dilettantes justement (et, parmi ces morceaux, les Douze mélodies semblent être celui qui a été reçu le plus bienveillamment). Ce qui à son tour pourrait expliquer l'oubli qui a englouti cet auteur après sa mort.

Une fois complétée cette présentation rapide des deux auteurs, on peut maintenant se tourner vers le fond de la question. Tout de même, une observation s'impose avant de pouvoir poursuivre vers les démarches interprétatives subséquentes : il faut signaler que, à cause de la pénurie de la littérature secondaire concernant Cazaneuve et Coppée, il est ardu d'aligner les propos dans la discussion qui suit avec ce que je viens de dire à propos du réseau des textes possibles. En effet, le réseau est ici presque inexistant, dans le sens où je ne connais pas d'interprétation détaillée de ce texte, tout comme de cette mélodie, et il faudra donc se contenter du réseau, assez réduit, qui peut être établi à partir de mes propres considérations sur ces objets artistiques. Pourquoi donc s'obstiner et choisir un morceau méconnu ? J'ai déjà mentionné mes raisons plus haut, et j'ajoute ici qu'il s'agit, me semble-t-il, d'un compromis entre deux désirs contrastants : d'un côté, le souhait d'enquêter de plus près des pièces qui ont été oubliée au fil du temps, de l'autre la possibilité de bénéficier d'un réseau textuel articulé et riche. Dans ce cas, le premier impératif a vaincu - l'antidote à ce type de problème serait d'étudier d'une façon systématique une portion considérable des morceaux méconnus.

\section{INVOCATION SOUS EXAMEN}

Après cette petite mise en contexte de la mélodie, l'on peut maintenant passer à l'analyse proprement dite. Tout d'abord, nous transcrivons le texte complet du poème de Coppée (1892, p. 291-292) :

Enfant blonde aux doux yeux, ô rose de Norvège, Qu'un jour j'ai rencontrée aux bords du bleu Léman, Cygne pur émigré de ton climat de neige!

Je t'ai vue et je t'aime ainsi qu'en un roman,

5 Je t'aime et suis heureux comme si quelque fée Venait de me toucher avec un talisman.

Quand tu parus, naïve et d'or vivant coiffée, J'ai senti qu'un espoir sublime et surhumain

Soudain m'enveloppait de sa chaude bouffée.

10 Voyageur, je devais partir le lendemain ;

Mais tu m'as pris mon cœur sans pouvoir me le rendre, Alors que pour l'adieu je t'ai touché la main.

A ce dernier bonheur j'étais loin de m'attendre, 


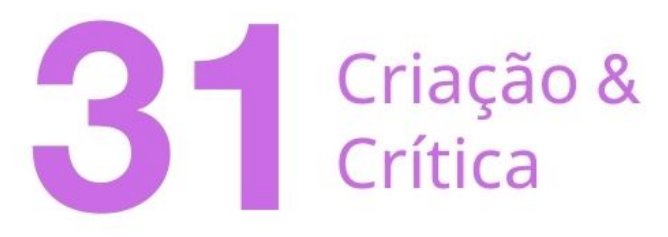

Et je me croyais mort à toutes les amours ;

Mais j'ai vu ton regard spirituel et tendre ;

Et tout m'a bien prouvé, dans les instants trop courts

Passés auprès de toi, blonde sœur d'Ophélie,

Que je pouvais aimer encore, et pour toujours.

Et je ne me dis pas que c'est une folie,

20 Que j'avais dix-sept ans le jour où tu naquis ;

Car le triste passé, je l'efface et l'oublie.

Et tu ne peux savoir à quel point c'est exquis !

Quelques remarques s'imposent à propos de l'organisation rimique, surtout lorsque l'on s'aperçoit que Cazaneuve n'a pas mis en musique le poème dans sa totalité - ce qui apporte des modifications considérables au schéma des rimes. On a donc ici une première fenêtre herméneutique, à savoir un point de sous-détermination engendrée par la suppression d'une partie du texte, qui demande une explication, ce que l'on essayera tout de suite.

Invocation est écrit en terza rima, une forme empruntée à l'Italie, et en particulier à la Divine Comédie de Dante, où, à cause de l'alternance des terminaisons, chaque tercet est accroché au suivant par sa rime orpheline (AQUIEN, 2018, p. 97, 106). Ceci appelle une solution irrégulière pour mettre fin à la succession rimique, qui pourrait idéalement se poursuivre à jamais - ce qui est exemplifié ici par le monostiche final, une conclusion qui n'est d'ailleurs pas à l'emploi exclusif de Coppée, et que l'on retrouve par exemple tout aussi bien dans les poèmes contemporains en terza rima de Verlaine ${ }^{21}$. Mais, comme j'ai anticipé plus haut, les coupures que Cazaneuve opère sur le texte à mettre en musique viennent perturber le schéma régulier des rimes ; en particulier, il est impossible de supprimer des tercets dans un poème en terza rima sans engendrer des rimes orphelines, ou, au moins, des rimes qui n'ont que deux instances en lieu des trois prévues. Cazaneuve supprime les tercets 4,5 , et 7 , tout comme le monostiche final. Par conséquent, la rime en -lie (v. 7) reste orpheline, tout comme la rime en -main du v. 8. Toutefois, cette dernière bénéficie de l'assonance avec la rime en -man des tercets précédents (v. 2, 4, et 6), et pourrait donc être comprise comme une quatrième instance irrégulière de la rime $\mathrm{B}$. La rime en -ours des v. 16 et 18, quant à elle, perd sa première instanciation (v. 14).

En revanche, Cazaneuve réalise des répétitions qui ne sont pas présentes dans le poème tel que Coppée l'a publié. Au niveau local, au v. 4, le syntagme « je t'aime » est répété deux fois à l'intérieur du vers, ce qui intensifie l'anaphore déjà mise en œuvre dans le texte par la reprise du vers suivant ; ensuite, le deuxième hémistiche du v. 7 est entièrement repris

\footnotetext{
${ }^{21}$ Pour une liste complète de ces poèmes, v. Aroui (1994, p. 113-114). En ce qui concerne la terza rima, " [son] histoire est comparable à celle du sonnet, et son cours à partir du milieu de la décennie 1830 résulte de la redécouverte des poètes du XVle siècle, même si la référence italienne est souvent mise en avant » (BURON, 2020, p. 279).
} 


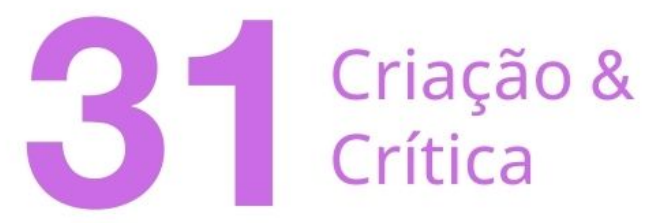

dans une exacerbation de l'emphase confiée à la ligne vocale 22 ; et finalement, au v. 18, le mot « encore », qui apparaît sans le e final dans l'édition Tellier, est répété à l'intérieur du vers, ce qui engendre de façon postiche un vers de quatorze syllabes. Au niveau global, en revanche, il n'y a qu'une reprise saillante : celle des deux premiers tercets à la fin de la mélodie. II s'agit d'un procédé assez commun et on le retrouve souvent chez d'autres compositeurs - il me semble qu'il signale ici simplement la conclusion imminente du morceau.

D'un point de vue formel, donc, la mélodie suit la forme $A B A$, où $A$ représente les deux premiers tercets, qui sont repris tels quels et dans la ligne vocale et dans l'accompagnement. A la fin du dernier A, Cazaneuve ajoute une petite coda, en modifiant la mise en musique du dernier hémistiche du v. $6^{23}$, qui est suivi par une mesure où l'on entend

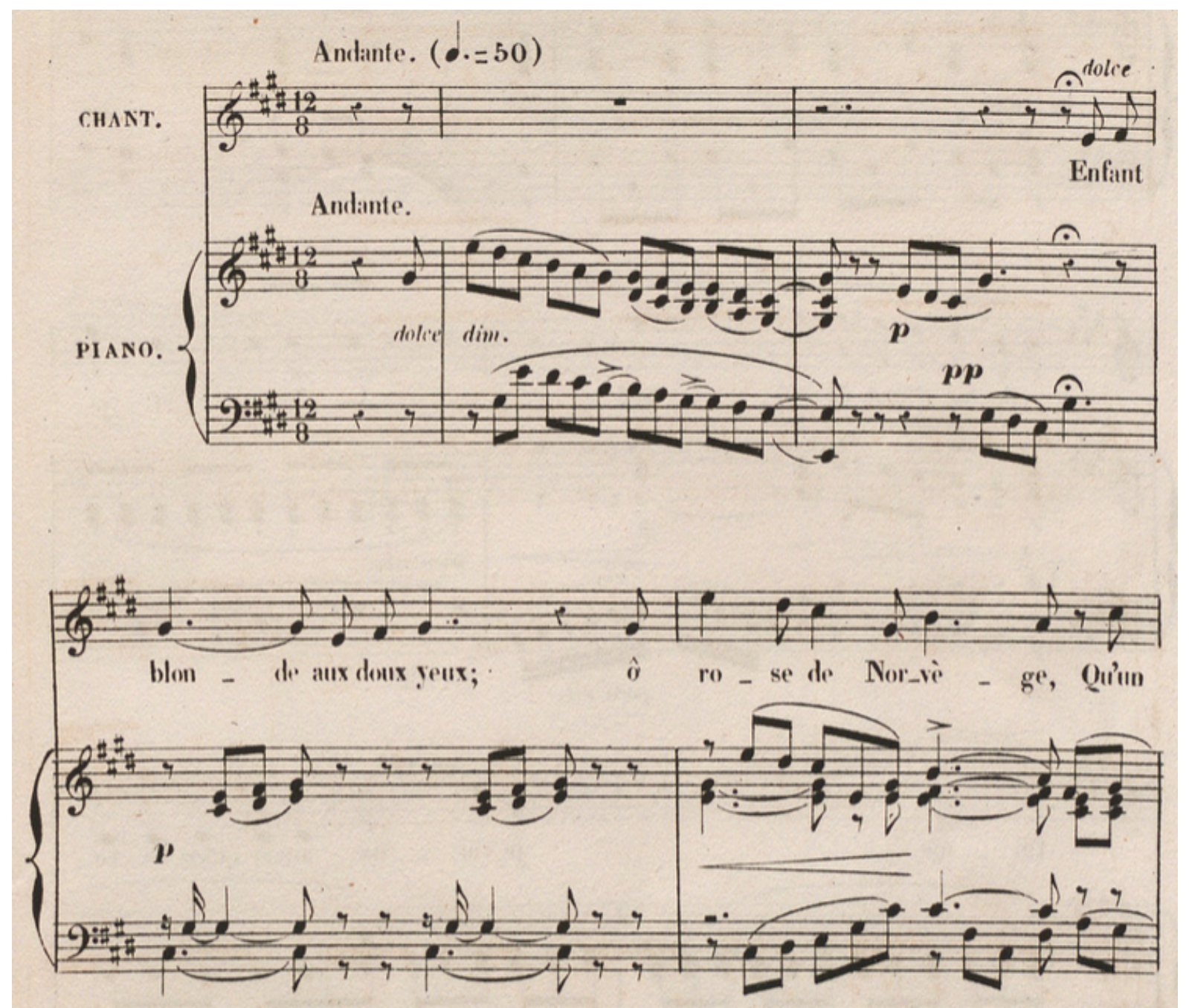

Ex. 1 : Edouard Cazaneuve (1879). Invocation, m. 1-5.

22 Aux m. 19-21, l'hémistiche " et d'or vivant coiffée » apparaît la première fois en correspondance d'une échelle descendante complète en do dièse majeur, alors qu'elle est répétée sur une harmonie de dominante, en montant de nouveau vers le registre aigu.

${ }^{23}$ La première fois que l'on entend la voix chanter ces mots, " avec un talisman », la ligne vocale est harmonisée par un accord de do dièse majeur, alors que, la deuxième fois, elle se termine sur un accord de do dièse mineur, qui sera prolongé jusqu'à la fin. 


\section{Criação \&
Crítica}

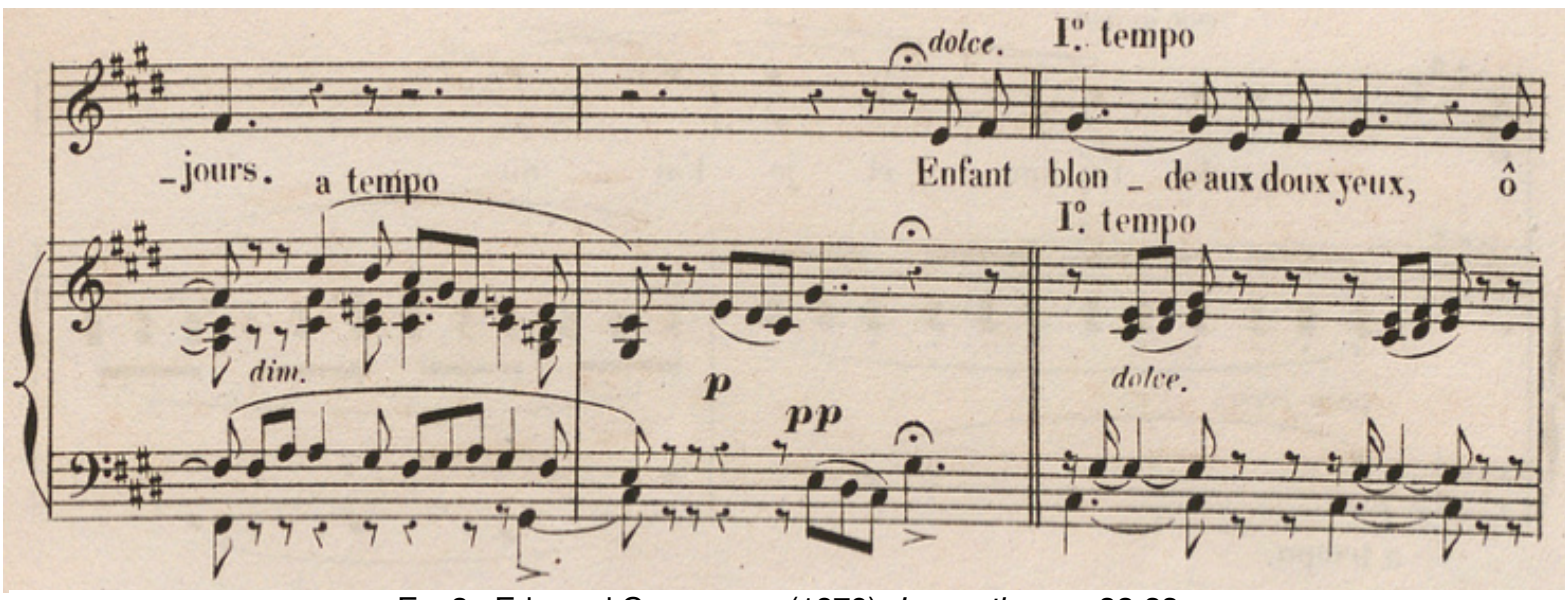

Ex. 2 : Edouard Cazaneuve (1879). Invocation, m. 32-33.

le piano seul. $B$, quant à lui, comprend les tercets 3 et 6 . Une ultérieure répétition au niveau macrostructural concerne, cette fois-ci, la partie de piano, qui introduit les deux parties A de manière similaire : dans les mesures 1 à 3 , tout au début, l'on entend une échelle descendante de do dièse éolien, qui présente le do dièse en tant que tonique du morceau malgré l'absence de la sensible ${ }^{24}$ (ex. 1) ; en revanche, lorsque la même échelle descendante est reprise dans les $\mathrm{m}$. 32 et 33, l'on retrouve l'altération accidentelle du septième degré, et toute ambiguïté est donc dissipée (ex. 2). L'on verra plus loin quelle est plausiblement la raison de cette différence.

Un dernier point à remarquer, relativement à l'architecture du poème, est constitué par les enjambements. L'on sait que ceux-ci se multiplient sous la plume de Coppée, dont les vers se trouvent ainsi marqués d'une qualité quelque peu prosaïque (MURPHY, 2000, p. 189, 203 ; GOUVARD, 1993, p. 46). Le premier que l'on rencontre en lisant le poème se vérifie entre les vers 5 et 6 : le sujet du verbe « venait », qui se trouve au début du v. 6 , figure à la fin du vers précédent. En dépit du fait que la relation syntaxique impliquée dans ce phénomène est assez contraignante, Cazaneuve insère tout de même des pauses entre les deux vers (ex. 3). S'il est vrai que la durée de ces dernières n'est pas suffisamment longue pour créer des problèmes de compréhension pour les auditeurs, il est indéniable que, au moins ici, l'enjambement ne perturbe pas la disposition des vers dans leur mise en musique - l'on pourrait peut-être imaginer une fenêtre herméneutique qui reste fermée. A l'inverse, l'enjambement qui se vérifie entre les v. 16 et 17, où le référent du participe passé " passés 》 (v. 17), à savoir « instants ", se trouve au v. 16, cause une modification dans l'organisation

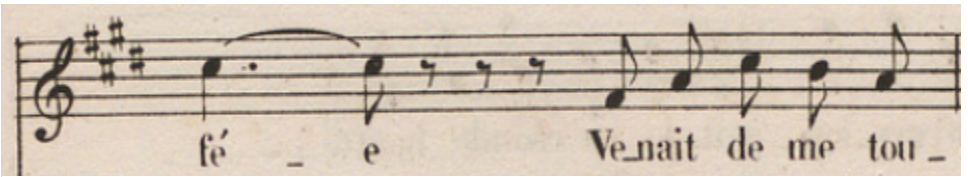

Ex. 3 : Edouard Cazaneuve (1879). Invocation, m. 16 (chant).

24 II serait peut-être possible de considérer ce passage comme un simple mi majeur se terminant sur un accord de tonique en premier renversement, surtout si l'on considère que, à partir des $\mathrm{m}$. 6 et 7 , le mi majeur devient évident. Toutefois, il me semble que ces premières mesures, jusqu'à m. 4 au moins, soient clairement centrées sur le do dièse, en particulier parce qu'il s'agit justement du début du morceau et il n'y a pas par conséquent un contexte préalable qui pourrait pousser l'écoute dans la direction du mi majeur. 


\section{Criação \&}

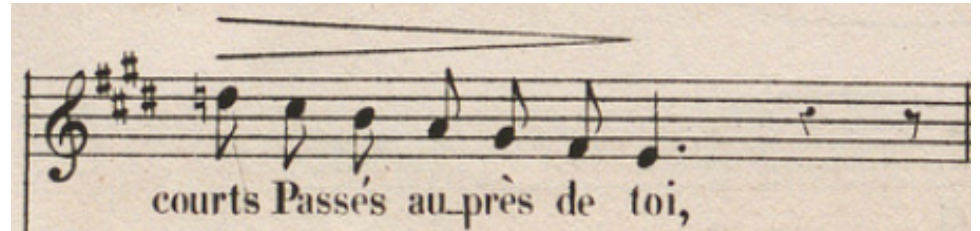

Ex. 4 : Edouard Cazaneuve (1879). Invocation, m. 28 (chant).

métrique des vers: en effet, le dernier hémistiche du v. 16 et le premier du v. 17 sont joints ensemble dans un alexandrin pour ainsi dire inédit, qui crée à ses deux côtés des vers de six syllabes (ex. 4). Ce type d'occurrence a pour effet de neutraliser l'une des fonctions de l'enjambement, c'est à-dire la mise en relief du premier vers concerné, qui reste ici largement inaperçue. En revanche, le rapprochement des vers intéressés, une fonction ultérieure de l'enjambement, est ici intensifié à l'extrême.

En ce qui touche au contraire à l'organisation structurale de la mélodie, j'ajoute une dernière observation. J'ai proposé plus haut de considérer ce morceau comme arrangé en $A B A+C$. II faut maintenant compléter cette description par une précision ultérieure à propos de $B$ : en effet, cette section peut être à son tour divisée en deux sous-sections, dont le point de raccord se trouve à la $\mathrm{m}$. 24, en correspondance de l'indication agogique «Animato » et du mot «Soudain ». J'ai également noté que B comprend les tercets 3 et 6 , et si l'on s'attend de trouver des sous-sections en correspondance de l'articulation strophique, l'on sera déçu, car les sous-sections, que l'on appellera désormais $\mathrm{B} 1$ et $\mathrm{B} 2$, se révèlent asymétriques en ce sens : B1 s'étend sur les v. 7 et 8 , alors que B2 embrasse les v. 9, 16, 17 et 18. L'on peut ainsi dresser un tableau complet de la structure d'Invocation.

Table 1 : Structure d'Invocation.
A
B1
B2
A
C
m. 1-18
m. $18-24$
m. 24-32
m. 33-47
m. 47-49
v. 1-6
v. $7-8$
v. $9 ; 16-18$
v. 1-6
v. 6
t. 1-2
t. 3
t. $3 ; 6$
t. 1-2
t. 2

Cette absence de symétrie peut paraître assez étrange compte tenu du fait que le $v$. 9 non seulement renferme un tercet mais présente également la dernière instance de la rime en -fée. Toutefois, une réflexion plus détaillée sur ce point permet de mieux comprendre une observation que j'ai faite plus haut, lorsqu'il était question des suppressions opérées par Cazaneuve sur le texte de Coppée : j'ai dit qu'il élimine les tercets 4, 5, et 7, et le monostiche suivant ce dernier. Je me suis déjà penché sur les conséquences que cette opération entraîne sur le schéma rimique, mais j'ai passé sur la question des effets sur l'organisation sémantique du poème. En général, ce texte est assez homogène : il se présente, comme son titre l'annonce, en tant qu'une invocation à la femme aimée, un genre qui n'est certainement pas nouveau dans la poésie lyrique ; son lexique ne donne pas de difficulté particulière ${ }^{25}$; et il serait difficile d'indiquer avec exactitude un moment de dénouement ou d'emphase particulière. Cazaneuve est présenté ainsi avec la possibilité de sélectionner librement le mot, le vers, le tercet, où ce point culminant se trouvera à l'intérieur de sa mélodie. Or, celui-ci est 


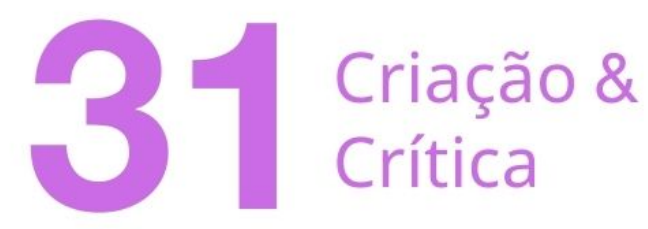

identifiable à l'entièreté de la sous-section B2, où, après le changement soudain de tempo, l'accompagnement se tait pendant le chant, qui se fait ainsi entendre seul jusqu'à la fin du segment (m. 32). En mettant en relation cette position de proéminence de B2 et les coupures qui l'intéressent, l'on s'aperçoit que certains traits de ces suppressions contribuent au sens de pathos qui caractérise cette partie. En particulier, dans le texte de Coppée, le v. 9 est suivi, au tout début du v. 10, par une apposition, qui précède à son tour une clarification sur la situation qui a causé l'apparition de l'amour. Il s'agit donc d'un moment de stagnation narrative : si des nouveaux éléments sont apportés au contexte, ceux-ci ne sont qu'un adjoint superflu au squelette du poème, lequel est déjà bien ébauché dans le premier tercet. En revanche, le v. 16, qui suit immédiatement le v. 9 dans la version remaniée par Cazaneuve, commence par la conjonction « et », dont l'effet est de projeter l'attention vers ce qui suit. Or, dans le texte de Coppée, cette conjonction est reprise en anaphore aux v. 19 et 22, ce qui configure ce dernier vers comme une augmentation de l'urgence émotive. Le compositeur, en effaçant le dernier tercet et le monostiche qui le suit, coupe court à la suite. Mais, par la répétition du mot " encor ", que l'on a déjà remarqué, il compense en créant un dénouement sur le mot " toujours », qui clôt ainsi B2 et qui est suivi par l'échelle descendante que l'on trouve au piano en guise d'introduction à la reprise de A. L'on comprend mieux maintenant la présence du si dièse, qui neutralise ici l'ambiguïté modale du début (ex. 2).

\section{Conclusion}

A la fin de cette analyse, on peut ajouter quelques remarques plus générales en considérant la mélodie Invocation comme l'endroit où l'interprétation du poème avancée par le compositeur se manifeste. Tout d'abord, si l'on envisage la forme métrique du poème et la narration d'un épisode d'amour comme deux éléments en tension, il me semble clair que Cazaneuve donne la priorité à ce dernier : si l'on observe les coupures qu'il apporte au texte, il est évident qu'il ne se soucie pas des caractéristiques propres à la forme strophique choisie par Coppée, et en particulier des enjeux spécifiques à la terza rima. En revanche, le compositeur ne perturbe jamais les occurrences toutes régulières des césures dans les alexandrins de Coppée, même quand un enjambement le pousse à rapprocher deux vers l'un de l'autre. Ce que l'on peut affirmer pour termine, c'est donc que Cazaneuve ressent comme contraignante la forme métrique des vers à un niveau microstructural, celui de l'articulation interne du vers lui-même, alors qu'il ne perçoit pas l'organisation strophique comme restrictive, et choisit dans tous les cas de privilégier la narration. Au-delà des limites de cet article, il serait intéressant de confronter la conduite du compositeur face au poème de Coppée avec ses autres mélodies, afin de constater s'il s'agit pour lui d'un procédé habituel ou, au contraire, d'une série des choix exceptionnels.

J'ai présenté ici un exemple isolé pour montrer le potentiel d'une conception littéraire de la mélodie française comme témoignage de la réception par les compositeurs des textes poétiques qu'ils choisissent pour leurs morceaux. Je souligne tout de même qu'il ne s'agit que d'une tentative qui pourra être améliorée et adaptée aux enjeux théoriques pertinents dans cet échange si nécessaire entre l'effort conceptualisant et la pratique interprétative. Je souhaite pour le moment que ma proposition puisse s'avérer intéressante et fructueuse en ce sens, et 


\section{Criação \&}

je mets en évidence que mon travail là-dessus n'est qu'à ses débuts et se poursuit tout au long de mes recherches.

\section{Références}

AGAWU, K. Playing with Signs: A Semiotic Interpretation of Classic Music. Princeton: Princeton University Press, 1991.

ANGER, V. La Mélodie française et Victor Hugo : éléments pour une synthèse impossible. Communication au Groupe Hugo du 4 avril 2015. Disponible sur: http://groupugo.div.jussieu.fr/Groupugo/15-04-04anger.htm. Accès en : 18 avril 2021.

ANONYME. « Music Abroad. Paris ». Dwight's Journal of Music, 27 juin 1863.

ANONYME. « Paris et départements ». Le Ménestrel, 13 mars 1892.

AQUIEN, M. La Versification. Paris : Que sais-je ? 2018.

AROUI, J.-L. « Le Monostiche verlainien ». Revue Verlaine, n. 2, pp. 108-128, 1994.

BORIO, G. ; GARDA, M. (dir.). L'esperienza musicale. Teoria e storia della ricezionoe. Turin : EDT, 1996.

BOTTE, A. « Auditions musicales ». La Revue et Gazette musicale de Paris, 10 mai 1863.

BOUCOURECHLIEV, A. Le Langage musical. Paris : Fayard, 1993.

BURON, E. « 'Renouvelons aussi / Toute vieille pensée'. L'usage des poèmes du XVle siècle par les poètes romantiques (Hugo, Gautier) ". In : MONFERRAN, J.-C. ; VÉDRINE, H. (dir.). Le XIXe siècle, lecteur du XVIe siècle. Paris : Classiques Garnier, pp. 263-290, 2020.

CAZANEUVE, É. Les Échos du bal. Mazurka-caprice pour piano, op. 1. Paris : G. Flaxland, 1859.

CAZANEUVE, É. Les Esprits Folles : grande valse de concert, composée pour le piano par Édouard Cazaneuve. Paris : E. Challiot, 1863.

CAZANEUVE, É. Priola : polka pour piano, op. 45. Paris : E. Chatot, 1872.

CAZANEUVE, É. Sérénade pour piano, op. 46. Paris : Heugel, 1872.

CAZANEUVE, É. Berceuse pour piano, op. 48. Paris : E. Chatot, 1872.

CHARLES, M. Rhétorique de la lecture. Paris : Éditions du Seuil, 1977.

CHARLES, M. «Trois hypothèses pour l'analyse, avec un exemple ». Poétique, vol. 164, n. 4, pp. 387-417, 2010. 


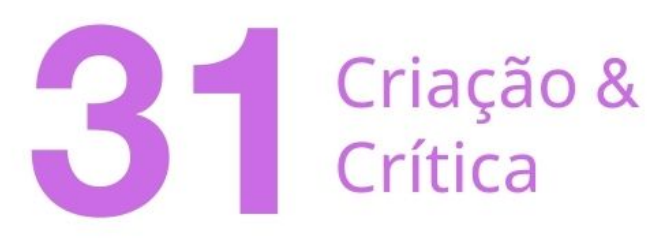

CONDEMI, C. Les Cafés-Concerts: histoire d'un divertissement, 1849-1914. Paris : Promeneur, 1992.

COPPÉE, F. CEuvres complètes de François Coppée, t. 2. Paris : L. Hébert, 1892.

COPPÉE, F. Chroniques artistiques, dramatiques et littéraires, éd. MORTELETTE, Y.. Paris : Presses de l'Université Paris-Sorbonne, 2003.

CORNULIER, B. de. Théorie du vers. Rimbaud, Verlaine, Mallarmé. Paris : Éditions du Seuil, 1982.

CORNULIER, B. de. Art poétique: notions et problèmes de métrique. Lyon: Presses Universitaires de Lyon, 1995.

DARCOURS, C. « Notes de musique ». Le Figaro, 26 décembre 1894.

DANCOURT. « Courrier de Paris ». La Gazette, 21 mars 1878.

DANCOURT. « Théâtres ». La Gazette, 2 mars 1881.

DEBUSSY, C. Ariettes oubliées. Paris : E. Fromont, 1903.

DELAGE, R. « Chabrier et Verlaine ». Revue Verlaine, n. 2, pp. 5-30, 1994.

FAUCHER, A.-M. La Mélodie française contemporaine: transmission ou transgression? Paris, L'Harmattan, 2010.

FAURÉ, G. 4 mélodies op. 51. Paris : J. Hamelle, 1888.

FISH, S. « Interpreting the 'variorum' ». Critical Inquiry, vol. 2, n. 3, pp. 465-485, 1976.

FISH, S. Quand lire c'est faire. L'Autorité des communautés interprétatives, trad. DOBENESQUE, E. Paris : Éditions Les Prairies Ordinaires, 2007.

GOUVARD, J.-. « Du vers classique au 12-syllabe de Verlaine ». Langue française, n. 99, pp. 45-62, 1993.

GOUVARD, J.-M. « Sur le statut phonologique de 'e' : la notion de 'e' féminin dans l'alexandrin de Verlaine ». Revue Verlaine, n. 2, pp. 87-107, 1994.

GRABOCZ, M. Sens et signification en musique. Paris : Hermann, 2007.

GRABOCZ, M. «Bref aperçu sur l'utilisation des concepts de narrativité et de signification en musique ». In : Musique, narrativité, signification. Paris : L'Harmattan, 2009.

GRIBENSKI, M. «Littérature et musique. Quelques aspects de l'étude de leurs relations ». Labyrinthe, pp. 111-130, 2004.

GRIBENSKI, M. Le Chant de la prose dans l'opéra (France, Italie, Allemagne), 1692-1902 : éléments de poétique, d'esthétique et d'histoire du goût. Thèse - Université Paris 4 Sorbonne, 2008. 


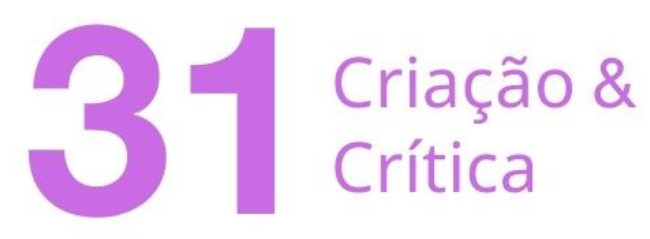

HANSLICK, E. Vom Musikalisch-Schönen. Ein Beitrag zur Revision der Ästhetik der Tonkunst, dritte verbesserte Auflage. Leipzig : Rudolph Weigel, 1865.

HATTEN, R. Musical Meaning in Beethoven: Markedness, Correlation, and Interpretation. Indianapolis : Indiana University Press, 1994.

JAUß, H.R. Pour une esthétique de la réception, trad. Claude Maillard. Paris : Gallimard, 1978.

JONCIĖRES, V. « Revue musicale ». La Liberté, 8 décembre 1879.

KRAMER, L. Critical Musicology and the Responsibility of Response: Selected Essays. Farnham : Ashgate, 2006.

KRAMER, L. Interpreting Music. Orlando : University of California Press, 2012.

KRAN. «Courrier des théâtres ». Le Réveil (Paris), 7 novembre 1881.

IMBERTY, M. «De quelques problèmes méthodologiques dans l'approche psychologique expérimentale de l'interprétation musicale ». In : DALMONTE. R. ; BARONI, M. (dir.). Secondo convegno europeo di analisi musicale. Atti. Trento: Università degli Studi di Trento, Diparimento di Storia della Civiltà Europea, 1992.

ISER, W. L'Acte de lecture. Théorie de l'effet esthétique, trad. Evelyne Sznycer. Bruxelles: Mardaga, 1985.

LANGER, S. Philosophy in a New Key. A Study in the Symbolism of Reason, Rite, and Art. Cambridge : Harvard University Press, 1942.

LEFRÈRE, J.-J. ; PIERSENS, M. (éd.). Les Têtes de Turc : Charles Baudelaire, les Belges, Edgar Bérillon, Émile Zola, François Coppée, Isidore Issou. Septième colloque des Invalides, 28 novembre 2003. Paris : Du Lérot, 2004.

LE MEUR, L. La Vie et l'œuvre de François Coppée. Paris : Éditions Spes, 1932.

MEYER, L.B. Emotion and Meaning in Music. University of Chicago Press, 1956.

MEYER, L.B. Explaining Music : Essays and Explorations. University of California Press, 1973.

MURPHY, S. «Éléments pour l'étude des 'Poèmes saturniens' ». Revue Verlaine, n. 3-4, pp. 165-274, 1996.

MURPHY, S. « Le Premier Verlaine : documents, variantes et exégèses ». Revue Verlaine, $\mathrm{n}$. 6, pp. 115-214, 2000.

NATTIEZ, J.-J. Musicologie générale et sémiologie. Paris : C. Bourgois, 1987.

PICORNOT, P. Six Poètes aux alentours du Musée Rodin, avec un choix de poèmes des six poètes présentés : Léon-Paul Fargue, François Coppée, Jean Loisy, René Char, Rainer Maria Rilke, Jean Cocteau. Paris : DL, 2010. 


\section{Criação \&}

Crítica

POPOVIC, P. «Les deux 'arts poétiques' de Paul Verlaine ». Études françaises, vol. 29, n. 3, pp. 103-121, 1993.

RATNER, L.C. Classic Music : Expression, Form, and Style. New York : Schirmer, 1980.

S.B. « Musique ». La Gazette, 14 janvier 1880.

THÉMINES, M. de. « Revue musicale ». La Patrie, 15 novembre 1880.

THÉMINES, M. de. « Revue musicale ». La Patrie, 21 février 1888.

VERHAEREN, É. «La Poésie ». L’Art moderne, 4 janvier 1891.

VERLAINE, P. Romances sans paroles. Paris : Léon Vanier, 1891.

VERLAINE, P. Les Hommes d'aujourd'hui. In : CEuvres complètes, t. V. Paris : Vanier, pp. 294299, 1905.

Recebido em: 23/08/2021

Aceito em: 21/11/2021

Referência eletrônica: PASINI, Lucia. La mélodie française comme témoignage d'une Réception : le cas d'invocation. Criação \& Crítica, n. 31, p., dez. 2021. Disponível em: $<\mathrm{http}: / /$ revistas.usp.br/criacaoecritica>. Acesso em: dd mmm. aaaa. 